\title{
DOWNSTREAM LOGISTICS PERFORMANCE MASTERY IN MOROCCAN AUTOMOTIVE INDUSTRY -INTER-ORGANIZATIONAL COORDINATION MASTERY APPROACH
}

\author{
CHOUAIB ELHAMMOUCHI \& ABDELLAH ABOUABDELLAH
}

Laboratory of System Engineering MOSIL, ENSA, University Ibn Tofail Kenitra, 14000 Morocco

\begin{abstract}
The purpose of this article is to propose the methodology of the construction of the logistic coordination interorganizational in the Moroccan automotive industry.

We will adopt an inductive procedure by beginning with a real situation analysis of setting up an interorganizational logistics coordination in the Moroccan automotive sector by highlighting the extent of the issue of interorganizational logistic coordination, then we will propose a theoretical model for this multi-stakeholder logistics coordination construction by taking into consideration the constraints related to Moroccan industry. We will explain later what these constraints are, the methodology to overcome them (rules and procedures), the coordination functioning once the constraints are managed.

KEYWORDS: Methodology, Logistics Coordination, Moroccan Automotive Sector \& Constraints
\end{abstract}

Received: Oct 21, 2019; Accepted: Nov 11, 2019; Published: Jul 21, 2020; Paper Id.: IJMPERDJUN2020402

\section{INTRODUCTION}

Measuring and evaluating logistics performance in companies and chains of companies in the Moroccan automotive sector is an essential way to maintain and develop the capacity of organizations to satisfy customers and ensure continuous improvement.

There are several professional repositories in this industry that evaluate and measure this performance [1]. These standards focus on the operational and / or strategic aspects of the company [2] without taking into consideration the global logistics chain. On the other hand, the control of the logistic performance is not relative only to the company itself.

In the Moroccan automotive industry, the absence of an inter-organizational coordination methodology is noted from the beginning of the process.

The absence of coordination between supplier-client (inter-organizational coordination) can lead to serious failures.

if the company wants to manage the downstream logistics performance, it must be aware of the need for coordination with its customers.

The Aim of this article is to propose an inter organizational coordination methodology that go through the identification and constraints treatment between companies, because and in general these upstream phases of these process put in place the structural framework in which the downstream phases will be ongoing. [3] They are 
manipulating potentials and concepts and produce upstream constraints; the more an activity is ongoing in downstream, the more it is free. The more an activity is ongoing in downstream, the more it generates huge number of constraints accumulated along the chain. in other terms the upstream create irreversibility's and downstream create residual reversibility's. [4]

\section{PROBLEMATIC}

The problem raised in this work relates to the definition of the inter-organizational logistics coordination approach and its adaptation to the specificities of the Moroccan company in the automotive sector.

\section{INDUSTRIAL CONTEXT: CASE OF AN INTER-ORGANIZATIONAL LOGISTICS COORDINATION BETWEEN A MOROCCAN EQUIPMENT MANUFACTURER AND ITS CUSTOMERS: ANALYSIS OF THE CONSTRAINTS}

The global automotive sector is experiencing a phase of significant growth with an increase in demand and supply to emerging countries. This new structuring of demand has forced investors to look for new locations to countries with a competitive global offer (proximity to large markets, logistics offer, skilled labor, etc.), to better adapt for customer needs.

In this new context, the Moroccan automotive industry is strengthening its position in the global automotive value chain, as evidenced by the remarkable performances recorded in recent years

- Automotive production exceeding 227570 vehicles in 2014 against only 18546 vehicles in 2003.

- 2nd producer in Africa after South Africa with a market share of 35\% in 2014 against 5\% in 2003

- Exports of this sector have crossed 40 billion dirhams in 2014.

Morocco is mainly present on the traditional value chain of the automotive sector, focusing on components and other inputs (suppliers 1st and 2nd row of the chain), as well as automotive assembly which has experienced a significant development in recent years with the implantation of the manufacturer Renault and PSA. In addition, the development of equipment manufacturers at $2 \mathrm{nd}$ and $3 \mathrm{rd}$ row is a major challenge that requires more effort from the companies that operate in this category in terms of the improvement

Automobile manufacturers still tending to externalize so many industrial activities to equipment specialist that need many customers.

In our case study there is a Moroccan equipment specialist of second rank, this subcontractor has decade of customer located around thousands of Kilometers.

The logistic coordination between this OEM have customer that goes through considering four cycles of transversal flows, which allows us to define four coordination phases as shown in the following diagram: 


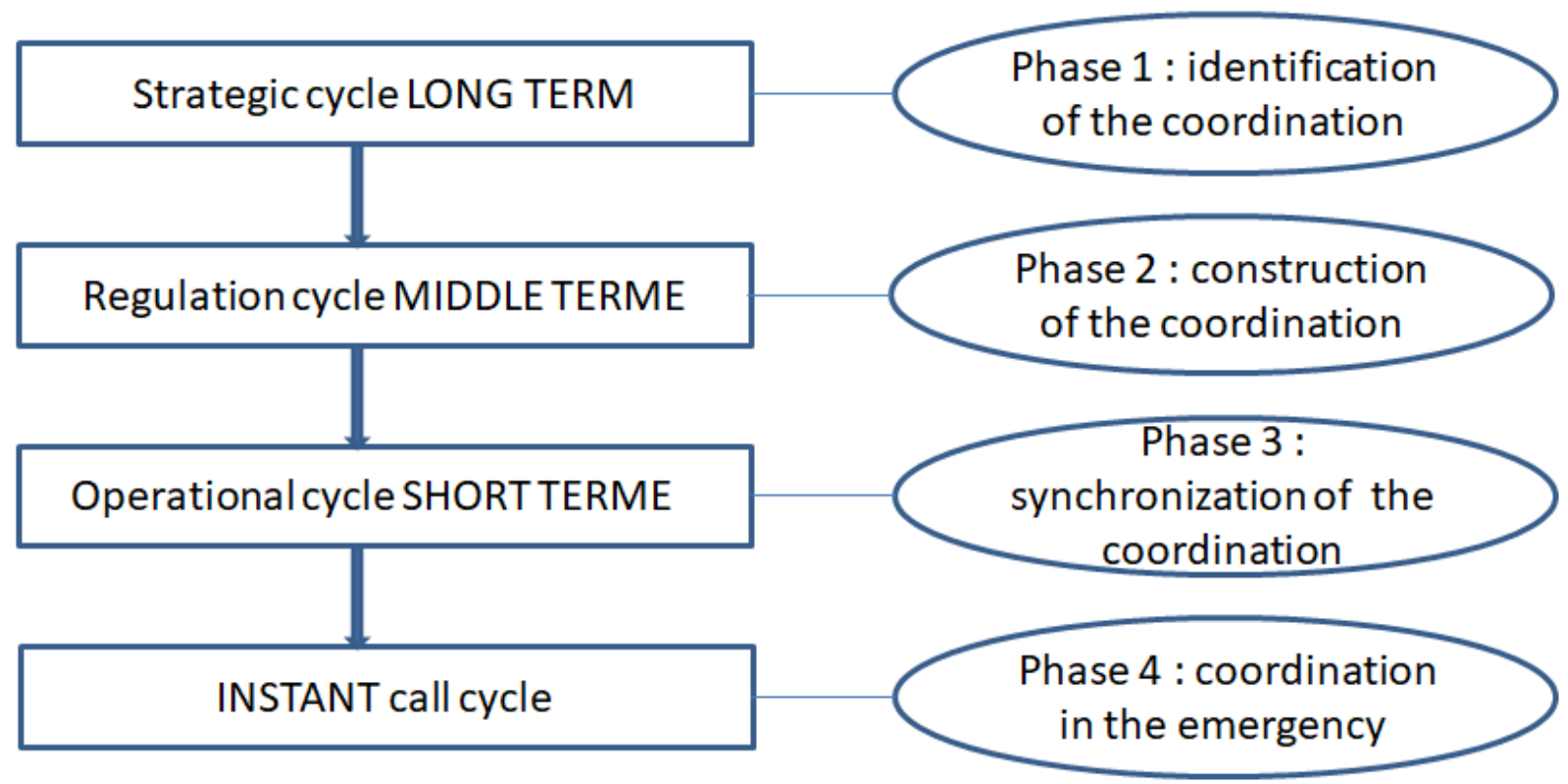

Figure 1: cycle of coordination

\subsection{Identification and Learning Phase of Constraints (Long Term)}

This phase, located in upstream coordination is elaborated in a long term, it is needed in prior to success future coordination.

The constraints that need to be taken into consideration when identifying this phase are:

\section{Identification of Cultural Constraints}

The national cultures difference between the EOM and its customers are the source of most of failures

Post coordination. beside difference between activity volume, customers are in most of cases big positions in Global level, in other side the Moroccan EOM are in a low position in National and international level.

We need also to add linguistics constraints, professional and competence ones.

\section{Identification of Organizational Constraints}

Before being engaged in coordination, customers start serial Audits at their suppliers to avoid any source of perturbation or dysfunction, these Audits touch financial aspects, structural, technology, Human resources, informatics, quality and logistics, with help of specified referential that are specific to automotive sector ( AMMOG/LE, SCOR, ASLOG, EVALOG)

- Structural Audit that define type of organization and heterogeneity of structures,

- Financial Audit to know better capital and financial capacity to ensure future Investments and extend activity.

- Technology and industrial Audit to define on which point technology, competence and process are controlled.

- Human Resources Audit to analyze social Policy coherence and homogeneity of future partnership.

- Informatically Audit to check aptitude capability of equipment to informatically integrate systems 
- Quality Audit by placing EOM under just in time conditions, to check production capacity to ensure daily deliveries of the orders. without omitting quality and lead time of products

- Logistics Audit [5] it considers evaluation of logistic organization, management, procurement logistics and suppliers, production organization evaluation, and distribution logistics evaluation.

In parallel of these Audits customer need to distribute to its partnership his targets and need also to support them to better understand how to work in a way to get a common evolution and achieve targets.

\section{Partnership Choice and Possibility of Simultaneous Engineering}

The contractual choice by the customer of his equipment manufacturer largely depends on the ability of the latter to support its evolutions and, more and more, its ability to engage in a simultaneous engineering process.

The supplier will then be involved from the outset in the development of a new vehicle model in terms of the technology that structures its business and that the manufacturer no longer masters: potential technological constraints will be identified and treated at source.

The following diagram summarizes the different types of constraints encountered in the strategic cycle in the Moroccan automotive sector:

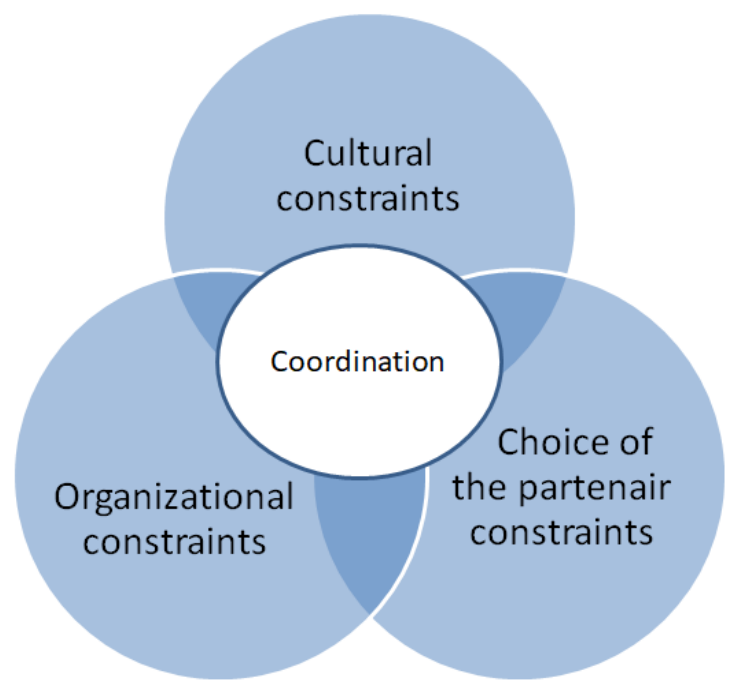

Figure 2:Inter-organizational constraints in the Moroccan automotive sector

\subsection{Construction Phase of the Coordination (In The Middle Term)}

The customer is entrusted with the realization of certain parts according to extremely rigorous specifications as well on the industrial and logistic plans (characteristics of the parts, rhythm of production, frequencies of deliveries etc....) in the organizational plan (procedure).

This midterm mean of regulation also deals with an agreement on computerized data exchange protocols that will allow the subsequent synchronization of processes; customer inform in a regular way his suppliers about production target with a quarterly plan, monthly and weekly: this short-term anticipation allows the equipment manufacturer to have the stocks of components that it needs. Finally, specific performance indicators (cost, timeliness, productivity, etc.) are put in place in such a way as to limit any divergence of interpretation on the results of the coordination. 


\subsection{The Synchronization Phase (Short Term)}

Call processes can then effectively synchronize the customer and vendor processes. In Our Example, every day, the customer issues orders in common language which is a delivery instruction specifying product references and quantities as well as delivery dates, after a second confirmation of loading message specifies the loading order of the truck that will deliver the products Of course, in logistic context, multiple hazards can disrupt the optimal course of operations: machine failure, traffic congestion etc... The traceability of flows is a necessity to identify these unforeseeable constraints.

\subsection{The Pase of the Coordination in the Emergency (Instant Process)}

The continuous monitoring of the Logistic process (tracing and tracking) makes it possible to instantly identify any failure.

Thus, in case of late delivery, for example, the duly informed client does not stop his production chain by seeking help from the supplier of production schedule change solutions, shipments of other goods in fast track (Hand carry, charter...).

Our study conducted in the Moroccan automotive sector allowed us then, to introduce 4 successive phases of the logistics coordination, control and control of the constraints of this coordination phase will lead to a mastery of the overall logistics performance of companies in the chain logistics.

\section{LOGISTIC COORDINATION INTERORGANIZATIONAL - CONSTRAINTS RESOLUTIONS}

The results obtained are inherent in the identification of inter-organizational coordination constraints that impact logistical performance.

If the constraints in the Long-term phase are well defined and resolved, the other phases will be easy to control.

First, constraints can be classified into two categories: explicit and implicit:

\section{Explicit Constraints}

Geographic: distance between the equipment manufacturer and its customers (hundreds of kilometers), travel time, time zones

Technical: Standardization, SCM technique, optimization technique, problem solving technique.

Technological: Integration capacity, openness to technology, computer system

Policy, Administrative and Regulatory: Stability, Security, Strategic Vision, Ease, Protection

Infrastructure: port, airport, free zone, highway

\section{Implied Constraints}

Cultural, Linguistic and Structural.

Now that the constraints are well defined on the different phases, we will propose a construction model of the interorganizational logistic coordination to avoid the problems in order to reach the optimal performance.

\section{Description and Model Operation}

The constitution of a multi-stakeholder logistic chain goes through a chronological succession in four stages each has an 
appropriate mode of communication:

First, we are faced with a set of constraints between actors (cultural, organizational, etc.). Logistical coordination therefore begins with the identification and understanding of the relevant constraints present (whether implicit or explicit) via an oral communication mode. -phase1- (long-term process).

Then a process of interaction leads the actors to define the rules of the game that they will lead: the confrontation of their logistical and organizational constraints with the implementation of performance indicators that allow to define the quality of their future coordination. -phase2- (Medium term process).

Therefore, it is a question of getting into the action of coordinating over time the operational system thus formed and of resulting in the synchronization of the logistic processes involved -phase3- (process Short term). This system needs inputs and generates outputs which in turn feedback on the quality of coordination defined in phase 2.

Finally -phase4-, multiple hazards can disrupt the optimal course of operations of the phase 3.

In case of a malfunction of the operational system, the supply chain is threatened. Logistic coordination accepts these constraints, these crises, but on the condition of detecting them immediately. Flow traceability is therefore a necessity to identify these unforeseeable constraints.

The inconsistent / outlier information detected at this stage will be cascaded to the decision chain. (Long / medium / short term process) to integrate it into the regular coordination.

The diagram below describes how this model works:

\begin{tabular}{|c|c|}
\hline Phase 1 & Phase 2 \\
\hline Constraints coordination & Constraints coordination \\
\hline Long Term & Middle Term \\
\hline Explicit and implicit constraints & Knowledge of the constraints \\
\hline Resolution through a communication process & Implementation of operating procedures, \\
\hline on working methods, coding, & exception, regulation \\
\hline \multicolumn{2}{|l|}{ relationships between stakeholders } \\
\hline & Distribution of responsibilities \\
\hline & Choice of performance indicators \\
\hline Phase 4 & Phase 3 \\
\hline \multirow[t]{2}{*}{ Coordination in the emergency $\leftarrow$} & Synchronization \\
\hline & Short Term \\
\hline \multicolumn{2}{|l|}{ Offset of operation (quantity, quality, time) } \\
\hline & almost automatic coordination of \\
\hline & the flows of the operational system over time \\
\hline Escalation of inconsistent / outlier information & ("synchronous flows", just-in-time, quick response) \\
\hline \multicolumn{2}{|l|}{ from the operational system. } \\
\hline & Operation of the operational system \\
\hline \multirow[t]{2}{*}{ Resolution by triggering an ALERT-BACKUP process } & defined in phase 2 \\
\hline & Perfect information \\
\hline
\end{tabular}

Figure 3: model of inter-organizational logistics coordination

\section{CONCLUSIONS}

We have built a model of inter-organizational logistics coordination in the Moroccan automotive sector. 
For that, we made an inductive reasoning: We started from the analysis of a real situation of interorganizational logistic coordination; we went back to the theory of the interorganizational logistic coordination in order to propose a general model of logistic coordination. It is advisable to continue the research around, then to validate it before integrating it sustainably in the logistic theory. The particularity of our approach is to propose a model of inter-organizational logistics coordination through the analysis of constraints related to the Moroccan automotive sector.

\section{REFERENCES}

1. Rim SGHIOURI and Chouaib ELHAMMOUCHI, Comparative study of Models measuring Logistic Supply chain performance in Moroccan automotive industry, Proceedings of the International Conference on Industrial Engineering and Operations Management Rabat, Morocco, April 11-13, 2017

2. Elasri, Khalid. "A LINGUISTIC AND CULTURAL ANALYSIS OF SYMPATHY, COMPASSION, EMPATHY AND PITY IN ENGLISH AND MOROCCAN ARABIC."International Journal of Linguistics and Literature (IJLL) 7. 4, Jun - Jul 2018; 81-90

3. Chouaib ELHAMMOUCHI, Impact of downstream logistics on the overall performance of the company - Case of a supplier in the automotive sector, CIE47 Proceedings, 11-13 October 2017, Lisbon / Portugal

4. Elenwo, E., and V. A. Akujuru. "THE EXPERT VALUERS VIEWS ON THE CRITICAL VARIABLES INFLUENCING RESIDENTIAL PROPERTY VALUES IN PORT HARCOURT METROPOLIS. "International Journal of Humanities and Social Sciences (IJHSS) 7. 4, Jun - Jul 2018; 193-204

5. Jacques COLIN, Diane FARAH, IMRL 2000 Third International Meeting for Research in Logistics Trois-Rivières, May 9, 10 and 112000

6. Bernard, Mushirabwoba, et al. "Preliminary Study Of Frictional Power Losses In Spur Geared Transmissions." International Journal 7 (2017): 199-208.

7. Philippe Lorino, Le deploiement de la valeur par les processus, $R F G$, juin-juillet-aout 1995, $p 65$.

8. EL HAJ, B. A. I. T., A. Bouayad, and M. Alami. "Quantitative evaluation of shrinkage porosity in alsi 9 cast alloy as function of titanium addition and casting parameters. "International Journal of Mechanical and Production Engineering Research and Development (IJMPERD) 8. 6, Dec 2018, 365-372

9. Source : Aurifeille et al., 1997, p87-88

\section{LIST OF FIGURES}

Figure 1: cycle of coordination

Figure 2:Inter-organizational constraints in the Moroccan automotive sector

Figure 3: model of inter-organizational logistics coordination 

